\title{
表面钒修饰对 $\alpha-\mathrm{Fe}_{2} \mathrm{O}_{3}$ 材料光电化学性能的增强作用
}

\author{
姚利珍 孔德生* 杜玖瑶 王 泽 张经纬 王 娜 李文娟 冯媛媛 \\ (曲阜师范大学化学与化工学院, 山东曲阜 273165)
}

\begin{abstract}
摘要: 对半导体材料进行表面化学修饰或改性, 是提高其光催化活性、有效利用光能的一种重要措施. 本文 结合水热化学法、化学池沉积和后续热处理等, 分别制备了未修饰 $\alpha-\mathrm{Fe}_{2} \mathrm{O}_{3}$ 和钒修饰的 $\alpha-\mathrm{Fe}_{2} \mathrm{O}_{3}$ 光电极材料. 利 用X射线粉末衍射(XRD)谱和紫外-可见漫反射光谱(UV-Vis-DRS)技术分析表征了材料的晶相结构、化学组成 和光谱吸收等固体物理化学性能; 利用光电流测量和电化学交流阻抗谱(EIS)实验技术, 并基于 $1 \mathrm{~mol} \cdot \mathrm{L}^{-1}$ $\mathrm{NaOH}$ ( $\mathrm{pH}$ 13.6)中的光电化学水分解反应, 研究了钥修饰对 $\alpha-\mathrm{Fe}_{2} \mathrm{O}_{3}$ 材料光电化学性能的增强作用. 结果表明, 与未修饰的 $\mathrm{Fe}_{2} \mathrm{O}_{3}$ 材料相比, 钒修饰 $\alpha-\mathrm{Fe}_{2} \mathrm{O}_{3}$ 样品出现 $\mathrm{FeVO}_{4}$ 的XRD特征峰, 但临界光吸收波长未发生红移; 钒 修饰使 $\mathrm{Fe}_{2} \mathrm{O}_{3}$ 材料的光电流增大 $4-5$ 倍、光生载流子在电极表面的复合几率降低了 $3 / 4-4 / 5$ 、电极表面电荷传 递速率 (表观一级速率常数) 明显提高. 结合 $\mathrm{Fe}_{2} \mathrm{O}_{3}$ / 溶液界面半导体能带模型和有关研究结果, 分析了研究体系 的界面电荷动力学传输过程以及钒修饰使 $\alpha-\mathrm{Fe}_{2} \mathrm{O}_{3}$ 材料光电化学性能增强的原因.
\end{abstract}

关键词：水分解；光生电荷传输；表面复合；光电流；交流阻抗谱

中图分类号: 0649

\section{Enhancement of the Photoelectrochemical Activity of $\alpha-\mathrm{Fe}_{2} \mathrm{O}_{3}$ Materials by Surface Modification with Vanadium}

\author{
YAO Li-Zhen \\ KONG De-Sheng* \\ DU Jiu-Yao \\ WANG Ze \\ WANG Na \\ LI Wen-Juan \\ FENG Yuan-Yuan \\ (Department of Chemistry and Chemical Engineering, Qufu Normal University, Qufu 273165, Shandong Province, P. R. China)
}

\begin{abstract}
Surface modification of semiconductor materials is an effective way to improve their photocatalysis and photo-conversion activities. Bare and $\mathrm{V}$-modified $\alpha-\mathrm{Fe}_{2} \mathrm{O}_{3}$ photoelectrode materials were prepared using hydrothermal, chemical bath deposition and heat treatment approaches. Their physicochemical and photoelectrochemical (PEC) properties were then investigated with X-ray diffractometry (XRD), UV-Vis diffuse reflectance spectroscopy (UV-Vis DRS), voltammetry, and electrochemical AC impedance spectroscopy (EIS) techniques. The existence of $\mathrm{FeVO}_{4}$ was indicated by its characteristic X-ray diffractometry patterns, while no significant red shifts in the photoabsorption edge were detected in UV-Vis diffuse reflectance spectroscopy spectra. With $\mathrm{V}$-modified and bare $\mathrm{Fe}_{2} \mathrm{O}_{3}$ serving as a photoanode, photoelectrochemical measurements were carried out for water splitting in $1 \mathrm{~mol} \cdot \mathrm{L}^{-1} \mathrm{NaOH}(\mathrm{pH} 13.6)$. The enhancement of $\alpha-\mathrm{Fe}_{2} \mathrm{O}_{3}$ photoelectrochemical activities through $\mathrm{V}$-modification was indicated by significantly increased photocurrents and decreased photocharge-recombination probability. By measuring electrochemical AC impedance spectroscopy spectra, pseudo-first-order rate constants for the charge transfer at the illuminated electrode/solution interface were estimated. The rate constant for $\mathrm{V}$-modification of the $\mathrm{Fe}_{2} \mathrm{O}_{3}$
\end{abstract}

Received: July 10, 2015; Revised: September 4, 2015; Published on Web: September 7, 2015.

*Corresponding author. Email: kongdscn@eyou.com; Tel: +86-537-4453069.

The project was supported by the Natural Science Foundation of Shandong Province, China (ZR2010EM026) and National Training Programs of Innovation and Entrepreneurship for Undergraduates, China (201410446044).

山东省自然科学基金(ZR2010EM026)和国家级大学生创新创业训练计划项目(201410446044)资助

CEditorial office of Acta Physico-Chimica Sinica 
electrode was higher than that of the bare $\mathrm{Fe}_{2} \mathrm{O}_{3}$ electrode. Improved interfacial charge transfer kinetics through $\mathrm{V}$-modification is responsible for the enhanced photoelectrochemical activities of $\alpha-\mathrm{Fe}_{2} \mathrm{O}_{3}$. The interfacial photocharge transfer and recombination processes and their properties are discussed with a semiconductor energy band model constructed for the electrode system.

Key Words: Water splitting; Interfacial charge transfer; Surface recombination; Photocurrent; AC impedance spectroscopy

\section{1 引言}

氢气具有高储能无污染的特点, 被认为是传 统化石能源的最佳替代品. 利用太阳能实现大规模 光电化学(PEC) 分解水制氢( 将太阳能转化为化学 能), 是国内外化学与材料研究者共同努力的一个目 标. ${ }^{1,2}$ 半导体光电极是PEC分解水制氢系统中的核心 构件. 金属氧化物半导体材料(如 $\mathrm{TiO}_{2} 、 \mathrm{Fe}_{2} \mathrm{O}_{3}$ 、 $\mathrm{WO}_{3} 、 \mathrm{ZnO}_{2} 、 \mathrm{SrTiO}_{4} 、 \mathrm{BiVO}_{4}$ 等)具有稳定性高、价 格低廉、绿色环保、易于制备等特点, 是目前光电 化学研究中备受关注的光电极基本材料. ${ }^{3-5}$

与其它常用的 $n$ 型半导体氧化物材料相比, $\alpha$ $\mathrm{Fe}_{2} \mathrm{O}_{3}$ 具有较适宜的禁带宽度 $\left(E_{\mathrm{g}}=2-2.2 \mathrm{eV}\right)$, 可吸收 波长小于 $550-600 \mathrm{~nm}$ 的太阳光(光子吸收近 $40 \%$ ), AM 1.5光照条件下的理论光电转化效率可达 $12.9 \%$, 被视为最具实际应用前景的光电极材料之一. $3^{3-6}$ 但 由于 $\alpha-\mathrm{Fe}_{2} \mathrm{O}_{3}$ 自身具有较低的电子迁移率 $\left(10^{-2}-10^{-1}\right.$ $\left.\mathrm{cm}^{2} \cdot \mathrm{V}^{-1} \cdot \mathrm{s}^{-1}\right)$ 、较短的空穴扩散长度 $(2-4 \mathrm{~nm}) 、$ 较慢 的表面析氧速率等不足, 致使光生载流子本体复合 及表面复合严重, 实际光电转化效率远低于理论值, 严重制约着 $\alpha-\mathrm{Fe}_{2} \mathrm{O}_{3}$ 作为光电极材料的实际应用.

为提高 $\alpha-\mathrm{Fe}_{2} \mathrm{O}_{3}$ 材料的PEC活性, 近几年来人们 在以下三个方面开展了大量研究工作: 一是制备纳 米结构的 $\alpha-\mathrm{Fe}_{2} \mathrm{O}_{3}$ 材料, 如纳米管、纳米棒、纳米颗 粒等 $\alpha-\mathrm{Fe}_{2} \mathrm{O}_{3}$ 材料, $1,7-9$ 二是利用其它元素对 $\alpha-\mathrm{Fe}_{2} \mathrm{O}_{3}$ 进 行本体掺杂, 常见的掺杂离子(或原子)主要有 $\mathrm{Nb}^{5+}$ 、 $\mathrm{V}^{5+} 、 \mathrm{Ti}^{4+} 、 \mathrm{Zr}^{4+} 、 \mathrm{Al}^{3+} 、 \mathrm{Cr}^{3+} 、 \mathrm{Co}^{3+} 、 \mathrm{Ir}^{3+} 、 \mathrm{C} 、 \mathrm{Si} 、$ $\mathrm{S} 、 \mathrm{~N} 、 \mathrm{P} 、 \mathrm{~F}$ 等 , $^{1,9-14}$ 三是利用其它元素或半导体材 料对 $\alpha-\mathrm{Fe}_{2} \mathrm{O}_{3}$ 进行表面修饰, 已报道的表面修饰材料 包括 $\mathrm{Ag} 、 \mathrm{Pt} 、 \mathrm{C}$ 、氧化钴 $\left(\mathrm{CoO}_{x}\right)$ 、氧化铱 $\left(\mathrm{IrO}_{x}\right)$ 、磷 酸盐等. ${ }^{15-21}$ 有关研究结果表明, 对 $\alpha-\mathrm{Fe}_{2} \mathrm{O}_{3}$ 进行本体 掺杂和表面修饰是增强 $\alpha-\mathrm{Fe}_{2} \mathrm{O}_{3}$ 电极PEC活性的两种 有效途径，但二者的作用机制不同. 在本体掺杂过 程中, 局外离子或原子将作为替位或填隙离子嵌入 $\mathrm{Fe}_{2} \mathrm{O}_{3}$ 晶格, 从而使掺杂 $\alpha-\mathrm{Fe}_{2} \mathrm{O}_{3}$ 材料的本体电子性能 或光吸收性能得到改善, 如(i) 提高了掺杂 $\mathrm{Fe}_{2} \mathrm{O}_{3}$ 材料 的本体电荷密度及电导率, 使光生载流子本体复合
受到抑制; ;,22,23 (ii) 禁带宽度减小, 增强了材料的可见 光吸收活性. ${ }^{13,24}$ 而表面修饰则对 $\alpha-\mathrm{Fe}_{2} \mathrm{O}_{3}$ 材料的表面 物理化学性能产生影响, 不同表面修饰材料对增强 $\alpha-\mathrm{Fe}_{2} \mathrm{O}_{3}$ 电极PEC活性的可能原因主要有以下几个方 面: (i) 电极的光谱吸收临界波长发生红移, 进一步 增强了可见光吸收活性:15 (ii) 存在表面等离子体共 振(SPR)增强效应, 促进了可见光吸收; ${ }^{25,26}$ (iii) 促进 了光生电子-空穴对的分离, 表面复合过程受到抑 制; ${ }^{20,21}$ (iv) 催化了光电极表面的水氧化反应, 提高了 表面/界面电荷传递速率等. ${ }^{2,19}$

由于 $\alpha-\mathrm{Fe}_{2} \mathrm{O}_{3}$ 具有较好的可见光吸收性能, 该氧 化物半导体材料不仅可用于太阳能分解水制氢(如 上所述), 而且可用于光催化降解环境有机污染 物, ${ }^{8,12,27}$ 或作为光吸收层用于对宽带隙半导体材料 (如 $\mathrm{TiO}_{2}$ ) 进行表面修饰. ${ }^{28-30}$ 因此, 研究高活性 $\alpha$ $\mathrm{Fe}_{2} \mathrm{O}_{3}$ 光电极材料的制备与性能, 将非常有助于促进 $\alpha-\mathrm{Fe}_{2} \mathrm{O}_{3}$ 材料在太阳能利用领域中的实际应用. 同时, 虽然有人研究过钒离子对 $\alpha-\mathrm{Fe}_{2} \mathrm{O}_{3}$ 光电极材料的本 体掺杂作用以及 $\mathrm{Fe}_{2} \mathrm{O}_{3}-\mathrm{FeVO}_{4}$ 二元体系的光催化或 化学催化作用, ${ }^{31-35}$ 但到目前为止, 文献中尚未见有 关钒对 $\alpha-\mathrm{Fe}_{2} \mathrm{O}_{3}$ 表面修饰作用的光电化学研究报道. 近期我们对 $\mathrm{TiO}_{2}$ 纳米管、 $\mathrm{Fe}_{2} \mathrm{O}_{3}$ 颗粒、棒状 $\mathrm{Ca}_{3} \mathrm{Bi}_{8} \mathrm{O}_{15}$ 等半导体材料的制备、光电化学分解水制 氢、光催化降解无机污染物、有关反应机理等方 面, 开展了部分研究工作, ${ }^{36-42}$ 本文中我们利用水热 化学法分别制备了 $\alpha-\mathrm{Fe}_{2} \mathrm{O}_{3}$ 和钒修饰 $\alpha-\mathrm{Fe}_{2} \mathrm{O}_{3}$ 光电极 材料, 并基于PEC水分解反应体系研究了钒修饰对 $\alpha-\mathrm{Fe}_{2} \mathrm{O}_{3}$ 光电化学活性的增强作用及原因.

\section{2 实验部分}

\section{1 钒修饰 $\alpha-\mathrm{Fe}_{2} \mathrm{O}_{3}$ 光电极材料的制备}

首先利用溶胶-凝胶法制备 $\mathrm{Fe}(\mathrm{OH})_{3}$ 胶体, 将 10 $\mathrm{mL} \mathrm{FeCl}_{3}$ 饱和溶液在搅拌条件下滴加至沸腾的 40 $\mathrm{mL}$ 蒸馏水中, 保持沸腾一定时间以使过多的水份蒸 发, 在半透膜中渗析-纯化, 得到棕红色 $\mathrm{Fe}(\mathrm{OH})_{3}$ 溶胶; 然后采用刮涂法将 $\mathrm{Fe}(\mathrm{OH})_{3}$ 胶体均匀地涂覆在钛片 
表面上, 控制膜厚 $100 \mu \mathrm{m}$ 、面积 $1 \mathrm{~cm}^{2}$, 并在马弗炉 中于 $550{ }^{\circ} \mathrm{C}$ 和大气条件下热处理 $1.5 \mathrm{~h}$, 制得 $\alpha$ $\mathrm{Fe}_{2} \mathrm{O}_{3}$ 光电极, 本文标记为“ $\mathrm{Fe}_{2} \mathrm{O}_{3}$ ”材料.

将制得的 $\mathrm{Fe}_{2} \mathrm{O}_{3}$ 电极分别放入 25 或 $90{ }^{\circ} \mathrm{C}$ 的 0.1 $\mathrm{mol} \cdot \mathrm{L}^{-1} \mathrm{VOSO}_{4}$ 溶液中浸渍 $1 \mathrm{~h}$, 然后在马弗炉中于 $550{ }^{\circ} \mathrm{C}$ 和大气条件下热处理 $1.5 \mathrm{~h}$, 得到钒修饰 $\mathrm{Fe}_{2} \mathrm{O}_{3}$ 电极, 本文分别标记为“ $\mathrm{V} @ \mathrm{Fe}_{2} \mathrm{O}_{3} 25{ }^{\circ} \mathrm{C}$ ”、 “V@ $\mathrm{Fe}_{2} \mathrm{O}_{3} 90^{\circ} \mathrm{C}$ ”材料. 为表征电极材料的晶相结构 和光学性能, 分别在相应条件下制备了 $\mathrm{Fe}_{2} \mathrm{O}_{3}$ 、 $\mathrm{V} @ \mathrm{Fe}_{2} \mathrm{O}_{3} 25^{\circ} \mathrm{C} 、 \mathrm{~V} @ \mathrm{Fe}_{2} \mathrm{O}_{3} 90^{\circ} \mathrm{C}$ 粉体材料. 所用溶 液均由分析级试剂和利用石英亚沸蒸馏器制得的 二次蒸馏水配制。光电极钛片基底(纯度 $99.7 \%$, Sigma-Aldrich)宽 $1 \mathrm{~cm}$ 、长 $6 \mathrm{~cm}$ 、厚度 $0.127 \mathrm{~mm}$, 使 用前进行表面打磨, 并在 $0.1 \mathrm{~mol} \cdot \mathrm{L}^{-1} \mathrm{HClO}_{4}+0.02$ $\mathrm{mol} \cdot \mathrm{L}^{-1} \mathrm{NaF}$ 溶液中浸泡清洗30 s.

\section{2 性能表征}

光电化学实验装置为 $\mathrm{H}$ 形双室三电极体系. 电 解池带有直径 $3.5 \mathrm{~cm}$ 的石英玻璃窗口, 以使入射光 垂直照射光电极表面, 以所制备光电极为光阳极(电 极表面 $\mathrm{Fe}_{2} \mathrm{O}_{3}$ 或 $\mathrm{V} @ \mathrm{Fe}_{2} \mathrm{O}_{3}$ 光活性层的面积 $1 \mathrm{~cm}^{2}$ )、铂 片为对电极、饱和甘录电极 $(\mathrm{SCE})$ 为参比电极, 电 解质溶液为 $1 \mathrm{~mol} \cdot \mathrm{L}^{-1} \mathrm{NaOH}$ 溶液 $(\mathrm{pH}$ 13.6). 实验仪 器包括CHI600d型电化学工作站(上海辰华仪器公 司)、AM 1.5太阳光模拟器(LSXS500, Zolix卓立汉 光), 入射光强度 $100 \mathrm{~mW} \cdot \mathrm{cm}^{-2}$. 光电流-电位 $\left(I_{\mathrm{ph}}-E\right)$ 曲线测量时的电位扫描速率为 $5 \mathrm{mV} \cdot \mathrm{s}^{-1}$, 光电 流-时间 $\left(I_{\mathrm{ph}}-t\right)$ 曲线测量时光照开/关转换频率为 0.05 $\mathrm{Hz}$, 电化学交流阻抗谱(EIS) 测量频率为 $1-10^{5} \mathrm{~Hz}$ (幅值 $10 \mathrm{mV}$ ). $I_{\mathrm{ph}}-E$ 曲线和 $E I S$ 谱分别在恒电位 $0.3 \mathrm{~V}$ (vs SCE) (即0.54 V (vs NHE(标准氢电极)) )下测量.

$X$ 射线衍射 $(X R D)$ 谱利用Miniflex 600型XRD粉 末衍射仪测量(Rigaku Co. Ltd., Japan), $\mathrm{Cu} K_{a}$ 辐射 (波长 $0.15406 \mathrm{~nm}$ ), 扫描速率为 $4^{\circ}(2 \theta) \cdot \mathrm{min}^{-1}$, 紫外-可 见漫反射光谱(UV-Vis-DRS)利用UV-3600型紫外/ 可见/近红外分光光度计测量(SHIMADZU Co., Japan), 积分球 $+\mathrm{BaSO}_{4}$ 背底, 扫描速率为 200 $\mathrm{nm} \cdot \mathrm{min}^{-1}$. EIS、XRD和UV-Vis-DRS测量结果分别 利用ZSimpWin程序、Jade软件包和KubelkaMunk函数分析处理. ${ }^{43-45}$

\section{3 结果与讨论}

\section{1 晶相结构与光吸收性能}

利用XRD粉末衍射技术表征了所制备 $\mathrm{Fe}_{2} \mathrm{O}_{3}$ 、
以及分别在 25 和 $90{ }^{\circ} \mathrm{C}$ 下所制备的钒修饰 $\mathrm{Fe}_{2} \mathrm{O}_{3}$ 样品 的晶相结构, 如图1所示. 由图1(a) 可见, 经过550 ${ }^{\circ} \mathrm{C}$ 热处理后的 $\mathrm{Fe}_{2} \mathrm{O}_{3}$ 为 $\alpha-\mathrm{Fe}_{2} \mathrm{O}_{3}$ (hematite) 晶型, 其特 征衍射峰(104)、(110)、(024)、(116)等与标准卡 JCPDS 33-0644 (图1(d)) 较好地吻合, 特征衍射峰峰 形尖锐, 说明材料的结晶程度较高. $\alpha-\mathrm{Fe}_{2} \mathrm{O}_{3}$ 样品的 平均晶粒度 $D$ 可利用最强衍射峰 (104) 并根据 Scherrer方程确定: ${ }^{46}$

$$
D=\frac{0.94 \lambda}{\beta \cos \theta}
$$

式中 $\lambda$ 为 $\mathrm{X}$ 光的入射波长, $\beta$ 为衍射峰的半峰宽 (FWHM), $\theta$ 为衍射角. 利用Jade软件所确定的(104) 晶面垂直方向上的平均晶粒度约为 $77 \mathrm{~nm}$.

钒修饰使 $\mathrm{Fe}_{2} \mathrm{O}_{3}$ 材料的XRD谱峰数量明显增多 (图1(b, c)). 分析表明, 在室温 $25^{\circ} \mathrm{C}$ 下所制备钒修饰 $\mathrm{Fe}_{2} \mathrm{O}_{3}$ 样品的XRD谱中(图 1(b)) 出现了 $\mathrm{FeVO}_{4}$ 的 (012)、(130)、(311)、(004)、(400)特征峰, 且与标 准卡JCPDS 89-0618 (图1(d))较好地吻合, 说明所形 成的 $\mathrm{FeVO}_{4}$ 为正交晶格结构; 而在 $90{ }^{\circ} \mathrm{C}$ 下所制备钒 修饰 $\mathrm{Fe}_{2} \mathrm{O}_{3}$ 样品的XRD谱中(图1(c))则进一步出现了 $\mathrm{FeVO}_{4}$ 的(112)和(040)谱峰. 含钒物种 $\mathrm{FeVO}_{4}$ 的存在, 证明了本文钒修饰 $\mathrm{Fe}_{2} \mathrm{O}_{3}$ 材料制备方法的有效性; $\mathrm{XRD}$ 结果同时表明, 在较高温度 $\left(90^{\circ} \mathrm{C}\right)$ 时所制备钒 修饰 $\mathrm{Fe}_{2} \mathrm{O}_{3}$ 材料中的 $\mathrm{FeVO}_{4}$ 晶相结构更加完整和典 型, 该结果与材料在光照条件下所表现出的不同光 电化学行为或性能较好地对应.

为探究钒修饰使 $\alpha-\mathrm{Fe}_{2} \mathrm{O}_{3}$ 材料光电化学活性增 强的可能原因, 进一步研究了三种光电极材料的光 学性能(如图2所示). 图2(a)是测量的可见光区的 UV-Vis-DRS谱, 图 $2 \mathrm{~b}$ 是根据Kubelka-Munk函数(式 (2)) 和Tauc方程(式(3))得到的 $[F(R) \cdot E]^{2}-E$ 曲线.

$$
\begin{aligned}
& F(R)=\frac{(1-R)^{2}}{2 R} \\
& {[F(R) \cdot h v]^{n}=A \cdot\left(h v-E_{\mathrm{g}}\right)}
\end{aligned}
$$

式中 $F(R)$ 为Kubelka-Munk函数; ${ }^{44,45} R$ 为漫反射系数; $h$ 为Plank 常数; $v$ 为入射光频率, $h v$ 为光子能量 $(\mathrm{eV})$; $A$ 为常数; $E_{\mathrm{g}}$ 为半导体禁带宽度; $n$ 为能带跃迁指数, $n=2$ 为直接跃迁, $n=1 / 2$ 为间接跃迁. 由图2(b)可以 看出, 钒修饰并未引起 $\mathrm{Fe}_{2} \mathrm{O}_{3}$ 材料禁带宽度的变化, 三种光电极材料的禁带宽度基本相同 $\left(E_{\mathrm{g}} \approx 2.05\right.$ $\mathrm{eV})$, 且三种 $\mathrm{Fe}_{2} \mathrm{O}_{3}$ 材料均为直接跃迁. 该结果表明, 钒对 $\alpha-\mathrm{Fe}_{2} \mathrm{O}_{3}$ 的表面修饰对材料本体光吸收性能的 

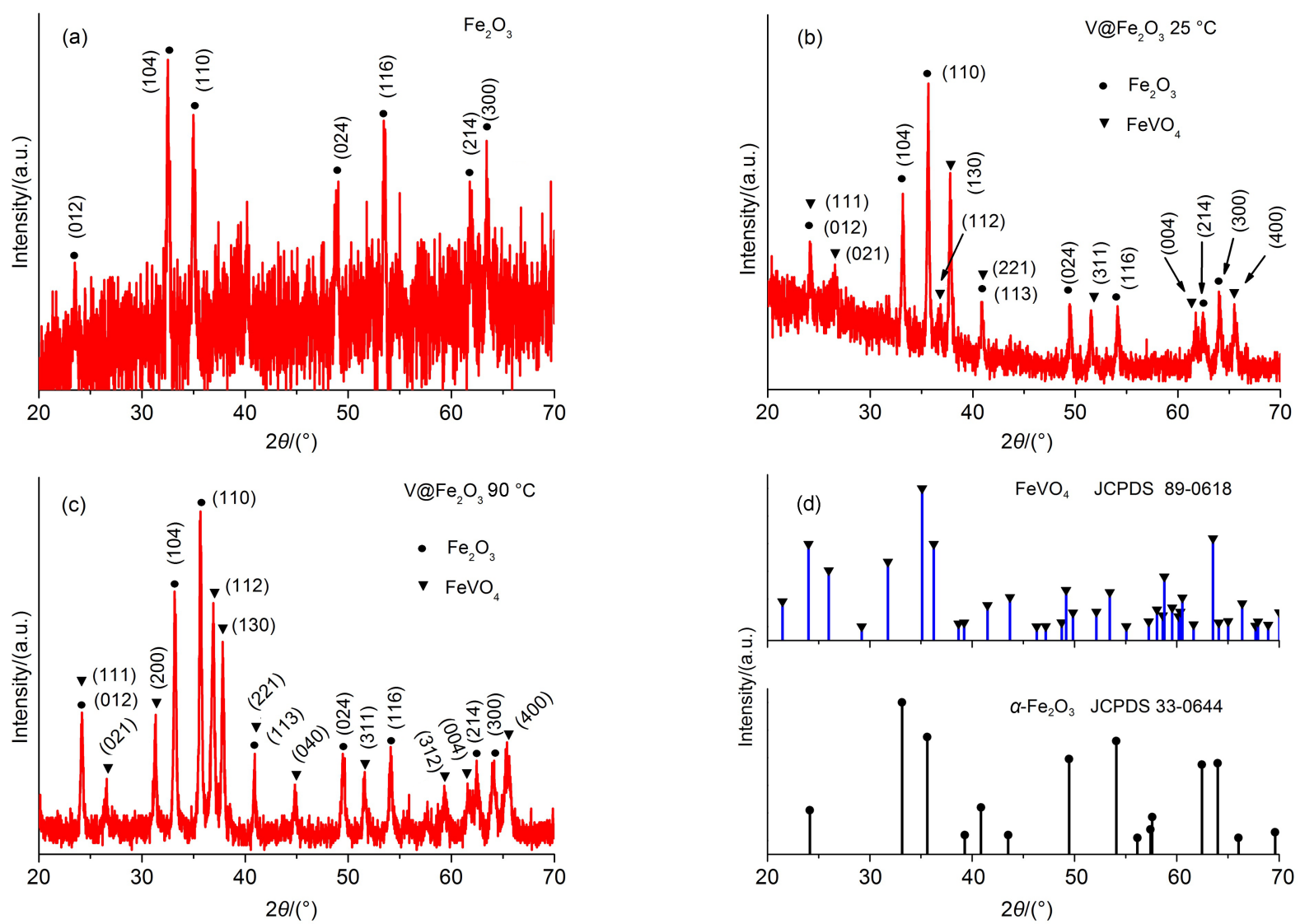

图1 光电极材料的XRD图谱

Fig.1 XRD patterns of the photoelectrode materials

(a) $\mathrm{Fe}_{2} \mathrm{O}_{3}$; (b, c) V-modified $\mathrm{Fe}_{2} \mathrm{O}_{3}$ at 25 and $90{ }^{\circ} \mathrm{C}$; (d) JCPDS card Nos. 33-0644 and 89-0618
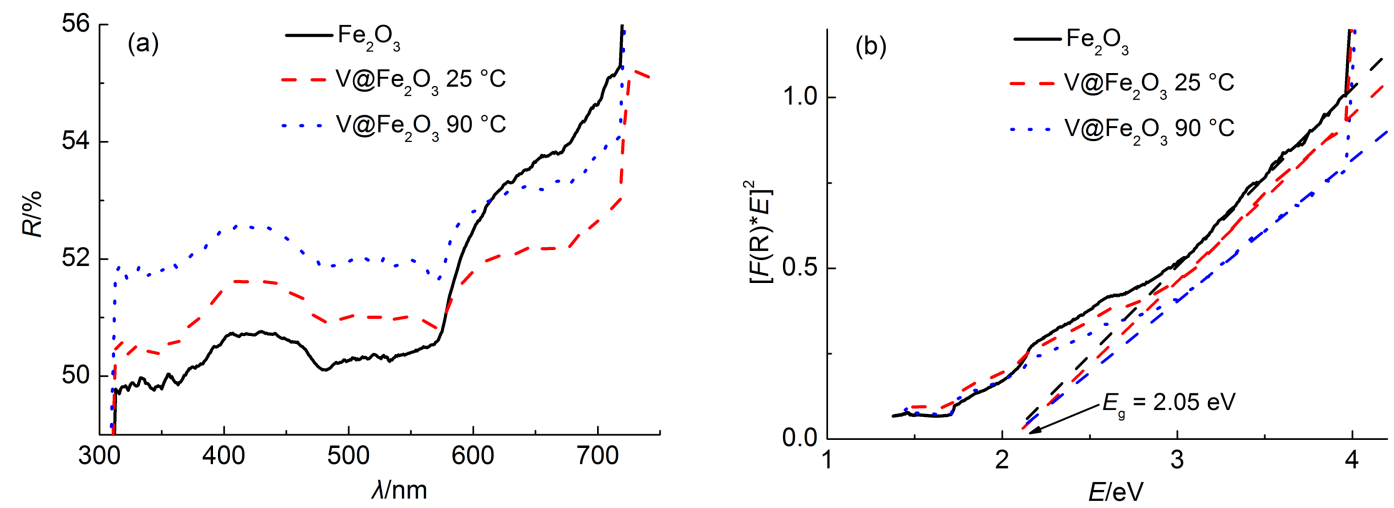

图2 $\mathrm{Fe}_{2} \mathrm{O}_{3}$ 及钒修饰 $\mathrm{Fe}_{2} \mathrm{O}_{3}$ 光电极材料的反射谱(a)与 $\mathrm{Tauc}$ 图(b)

Fig.2 Reflection spectra (a) and $\mathrm{Tauc}_{2} \mathrm{Fe}_{2} \mathrm{O}_{3}$ (b) of $\mathrm{Fe}_{2} \mathrm{O}_{3}$ and $\mathrm{V}$-modified $\mathrm{Fe}_{2} \mathrm{O}_{3}$ samples

$R$ : diffuse reflectance

影响很小, 不会引起材料临界光吸收波长的红移.

\section{2 光电流响应}

通过光电流测量研究了钒的表面修饰对 $\mathrm{Fe}_{2} \mathrm{O}_{3}$ 材料光电化学性能的影响. 图2是利用三电极 体系在 $1 \mathrm{~mol} \cdot \mathrm{L}^{-1} \mathrm{NaOH}(\mathrm{pH}$ 13.6)溶液中测量的
$I_{\mathrm{ph}}-E$ 曲线. 本研究所用电解质溶液为 $\mathrm{NaOH}$, 不含其 它电活性物种, 有关电极过程对应于水的光电化学 分解:

$$
\left.\mathrm{H}_{2} \mathrm{O} \stackrel{h v}{\rightarrow} \frac{1}{2} \mathrm{O}_{2} \text { (光阳极 }\right)+\mathrm{H}_{2}(\mathrm{Pt} \text { 阴极 })
$$

由图2可见，与未修饰的 $\mathrm{Fe}_{2} \mathrm{O}_{3}$ 电极相比，钒修饰 
$\mathrm{Fe}_{2} \mathrm{O}_{3}$ 电极的光电流明显增大, 且在 $90{ }^{\circ} \mathrm{C}$ 下钒修饰 $\mathrm{Fe}_{2} \mathrm{O}_{3}$ 样品的光电化学活性高于在室温 $25^{\circ} \mathrm{C}$ 下钒修 饰的 $\mathrm{Fe}_{2} \mathrm{O}_{3}$ 样品. 另外, 三种电极在无光照条件下的 暗电流很小, 在 $E<0.5 \mathrm{~V}(v s \mathrm{SCE})$ 电位范围内的暗 电流密度为 $10^{-7}-10^{-5} \mathrm{~A} \cdot \mathrm{cm}^{-2}$, 故可将光照条件下的 电流值视为光电流 $\left(I_{\mathrm{ph}}\right)$.

图3为恒电位 $0.3 \mathrm{~V}(v s \mathrm{NHE})$ 下的暂态 $I_{\mathrm{ph}}-t$ 转换 曲线. 由图 3 可见, 在光照瞬间光电流密度急剧上升 并达到峰值 $I_{\mathrm{ph}}(\max )$ (见图中标注), 然后逐渐降低达 到稳态. 经过 3 次光照开/关循环后, 光电流密度相应 逐渐趋于稳定, 三种电极材料在 $140 \mathrm{~s}$ 时的峰值光电 流密度 $I_{\mathrm{ph}}(\max )$ 和 $160 \mathrm{~s}$ 时的暂态光电流密度 $I_{\mathrm{ph}}(\mathrm{ss})$ 见 表1. 由表1可见, 与未修饰的 $\mathrm{Fe}_{2} \mathrm{O}_{3}$ 电极相比, 在室温 $\left(25{ }^{\circ} \mathrm{C}\right)$ 的含钒溶液中所制备的钒修饰 $\mathrm{Fe}_{2} \mathrm{O}_{3}$ 电极 $\mathrm{V} @ \mathrm{Fe}_{2} \mathrm{O}_{3} 25^{\circ} \mathrm{C}$ 的 $I_{\mathrm{ph}}(\mathrm{ss})=0.096 \mathrm{~mA} \cdot \mathrm{cm}^{-2}$, 约为未修 饰 $\mathrm{Fe}_{2} \mathrm{O}_{3}$ 的 3 倍; 在 $90{ }^{\circ} \mathrm{C}$ 的含钒溶液中所制备的 $\mathrm{V} @ \mathrm{Fe}_{2} \mathrm{O}_{3} 90^{\circ} \mathrm{C}$ 电极的 $I_{\mathrm{ph}}(\mathrm{ss})=0.14 \mathrm{~mA} \cdot \mathrm{cm}^{-2}$, 约为 未修饰 $\mathrm{Fe}_{2} \mathrm{O}_{3}$ 的4倍. 这表明, 钒修饰使 $\mathrm{Fe}_{2} \mathrm{O}_{3}$ 材料的光 电化学活性显著增强, 且在较高温度下 $\left(90^{\circ} \mathrm{C}\right)$ 所制 备钒修饰 $\mathrm{Fe}_{2} \mathrm{O}_{3}$ 材料的光电化学活性更高, 该结果与 $I_{\mathrm{ph}}-E$ 曲线测量结果(图2)相吻合.

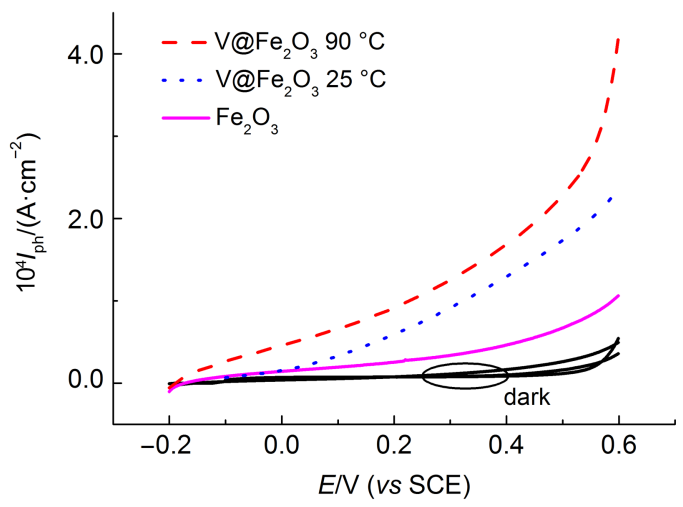

图3 $\mathrm{Fe}_{2} \mathrm{O}_{3}$ 及钒修饰 $\mathrm{Fe}_{2} \mathrm{O}_{3}$ 光电极的光电流密度-电位曲线

Fig.3 $I_{\mathrm{ph}}-E$ curves of $\mathrm{Fe}_{2} \mathrm{O}_{3}$ and $\mathrm{V}$-modified $\mathrm{Fe}_{2} \mathrm{O}_{3}$ photoelectrodes

$1 \mathrm{~mol} \cdot \mathrm{L}^{-1} \mathrm{NaOH}\left(\mathrm{pH} 13.6\right.$ ) aqueous solution; scan rate $v=5 \mathrm{mV} \cdot \mathrm{s}^{-1}$; AM $1.5\left(100 \mathrm{~mW} \cdot \mathrm{cm}^{-2}\right)$ incident light
光照开始后光电流密度由 $I_{\mathrm{ph}}(\max )$ 快速衰减为 $I_{\mathrm{ph}}(\mathrm{ss})$ (图3), 其原因主要是由于光生电子-空穴对在 “光电极/溶液”界面发生的表面复合过程所致. 因此, 钒修饰对光电极 $I_{\mathrm{ph}}-t$ 曲线(图4)暂态转换行为 的影响, 反映了钒修饰对光生载流子在 $\mathrm{Fe}_{2} \mathrm{O}_{3}$ 电极表 面复合过程的影响，并可用表观表面复合几率 (SPR)表征之: ${ }^{36}$

$$
\mathrm{SPR}=\frac{I_{\mathrm{ph}}(\max )-I_{\mathrm{ph}}(\mathrm{ss})}{I_{\mathrm{ph}}(\max )}
$$

根据式(5)和表 1 中的 $I_{\mathrm{ph}}(\mathrm{ss})$ 及 $I_{\mathrm{ph}}(\max )$ 数据所确定的 三种电极材料的表观复合几率列于表1中. 可以看 出, $\mathrm{Fe}_{2} \mathrm{O}_{3}$ 材料表面经过钒修饰后, 表观 SPR仅为未 修饰 $\mathrm{Fe}_{2} \mathrm{O}_{3}$ 材料的 $1 / 4-1 / 5$, 光生载流子的表面复合过 程受到明显的抑制. 由于光生导带 $(\mathrm{CB})$ 电子与价带 (VB)空穴之间的表面复合是对已吸收光子的直接 损耗, 因此该结果表明, 与未修饰的 $\mathrm{Fe}_{2} \mathrm{O}_{3}$ 电极相比, 钒修饰 $\mathrm{Fe}_{2} \mathrm{O}_{3}$ 电极对所吸收光子的有效利用率(即光 电转化效率)提高了约 $75 \%-80 \%$. 为进一步探究钒 修饰对 $\mathrm{Fe}_{2} \mathrm{O}_{3}$ 材料光电化学性能的增强作用(以及对 光生载流子表面复合过程的抑制作用)的原因, 本文

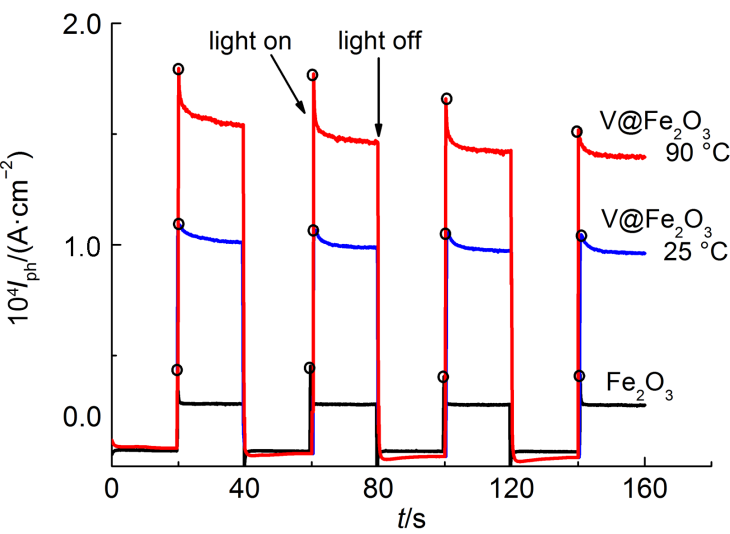

图4 $\mathrm{Fe}_{2} \mathrm{O}_{3}$ 及钒修饰 $\mathrm{Fe}_{2} \mathrm{O}_{3}$ 光电极的暂态光电流密度响应

Fig.4 Transient photocurrent density responses of $\mathrm{Fe}_{2} \mathrm{O}_{3}$ and $\mathrm{V}$-modified $\mathrm{Fe}_{2} \mathrm{O}_{3}$ photoelectrodes

$1 \mathrm{~mol} \cdot \mathrm{L}^{-1} \mathrm{NaOH}(\mathrm{pH} 13.6)$ aqueous solution; optenyial $E=0.3 \mathrm{~V}(v s$ $\mathrm{SCE}) ; \mathrm{AM} 1.5\left(100 \mathrm{~mW} \cdot \mathrm{cm}^{-2}\right)$ incident light

表1 $\mathrm{Fe}_{2} \mathrm{O}_{3}$ 与钒修饰 $\mathrm{Fe}_{2} \mathrm{O}_{3}$ 光电极的峰值光电流密度 $\left(I_{\mathrm{ph}}(\max )\right)$, 暂态光电流密度 $\left(I_{\mathrm{ph}}(\mathrm{ss})\right)$ 及载流子表面复合几率

Table 1 Values of peak photocurrent densities $\left(I_{\mathrm{ph}}(\max )\right)$ and transient photocurrent densities $\left(I_{\mathrm{ph}}(\mathrm{ss})\right)$, and apparent surface-recombination probability of charge-carriers for $\mathrm{Fe}_{2} \mathrm{O}_{3}$ and $\mathrm{V}$-modified $\mathrm{Fe}_{2} \mathrm{O}_{3}$ photoelectrodes

\begin{tabular}{cccc}
\hline Material & $I_{\mathrm{ph}}(\mathrm{ss}) /\left(\mathrm{A} \cdot \mathrm{cm}^{-2}\right)$ & $I_{\mathrm{ph}}(\mathrm{max}) /\left(\mathrm{mA} \cdot \mathrm{cm}^{-2}\right)$ & $\mathrm{SPR}^{\dagger} / \%$ \\
\hline $\mathrm{Fe}_{2} \mathrm{O}_{3}$ & $2.77 \times 10^{-5}$ & $4.09 \times 10^{-5}$ & 32.3 \\
$\mathrm{~V} @ \mathrm{Fe}_{2} \mathrm{O}_{3} 25^{\circ} \mathrm{C}$ & $9.63 \times 10^{-5}$ & $1.05 \times 10^{-4}$ & 8.29 \\
$\mathrm{~V} @ \mathrm{Fe}_{2} \mathrm{O}_{3} 90^{\circ} \mathrm{C}$ & $1.40 \times 10^{-4}$ & $1.51 \times 10^{-4}$ & 7.28 \\
\hline
\end{tabular}


利用交流阻抗技术研究了光照条件下电极/溶液界 面的电荷传输动力学性能.

\section{3 交流阻抗谱测量}

交流阻抗技术是研究各种电极/溶液界面电荷 传输动力学性能的重要手段. ${ }^{39,49}$ 图 5 为 $\mathrm{Fe}_{2} \mathrm{O}_{3}$ 、 $\mathrm{V} @ \mathrm{Fe}_{2} \mathrm{O}_{3} 25{ }^{\circ} \mathrm{C} 、 \mathrm{~V} @ \mathrm{Fe}_{2} \mathrm{O}_{3} 90{ }^{\circ} \mathrm{C}$ 三种光电极在1 $\mathrm{mol} \cdot \mathrm{L}^{-1} \mathrm{NaO}$ 溶液中、光照条件下测量的EIS谱. 由 图5可见, 三种光电极材料的界面阻抗响应均表现 为 2 个时间常数, 这与文献报道结果 ${ }^{11,50,51}$ 类似. 结合 文献中有关金属氧化物半导体材料(特别是 $\mathrm{TiO}_{2}$ 和 $\mathrm{Fe}_{2} \mathrm{O}_{3}$ ) 表面PEC析氧反应机理的阻抗研究, 18,36,37,38,50,52 高频时间常数可归因于光生空穴在 “光电极/溶液” 界面的Faraday电荷传输过程, 低频时间常数可归因 于阳极析氧过程中电极表面所形成的中间含氧物 种(如羟基自由基 $-\mathrm{OH}^{*}$ 或过氧化物 $-\mathrm{O}-\mathrm{O}-$ )的 吸/脱附过程. 图5同时给出了相应的等效电路, 其 $\mathrm{CDC}$ 描述码为 $R_{\mathrm{s}}\left(C_{\mathrm{dl}}\left(R_{\mathrm{ct}}\left(R_{\mathrm{a}} Q\right)\right)\right)$, 其中 $R_{\mathrm{s}}$ 为溶液电阻, $C_{\mathrm{dl}}$ 为电极/溶液界面 Helmholtz双层电容, $R_{\mathrm{ct}}$ 为界面 电荷传输电阻, $R_{\mathrm{a}}$ 是与中间物种吸脱附过程有关的 等效电阻, $Q$ 为常相位角元件 (CPE), CPE的阻抗为 $Z_{\mathrm{CPE}}=Y_{0}^{-1} \cdot(\mathrm{j} \omega)^{-n},{ }^{27,49}$ 其中 $Y_{0}$ 和 $n$ 分别是与频率无关的 $\mathrm{CPE}$ 参数.

图6为利用等效电路模型(图5)得到的拟合频谱 与测量频谱的Bode图, 可见二者基本吻合, 说明所 使用的等效电路模型能较好地描述电极表面的光 电化学过程, 计算确定的有关等效电路元件参数见

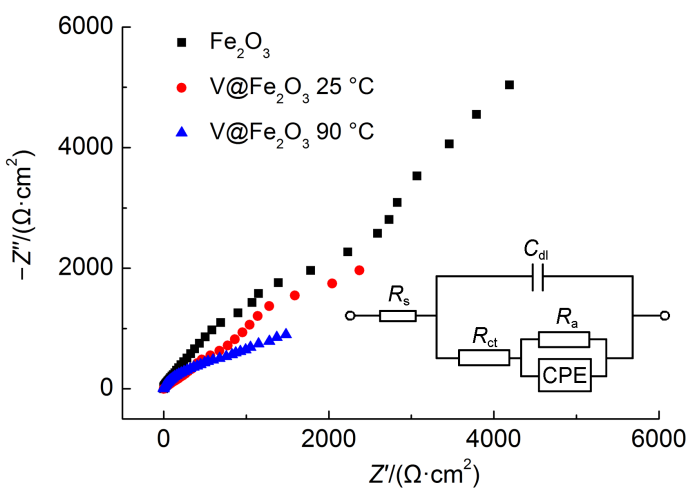

图5 $\mathrm{Fe}_{2} \mathrm{O}_{3}$ 及钒修饰 $\mathrm{Fe}_{2} \mathrm{O}_{3}$ 光电极的 EIS 图

Fig.5 EIS Nyquist plots of $\mathrm{Fe}_{2} \mathrm{O}_{3}$ and $\mathrm{V}$-modified $\mathrm{Fe}_{2} \mathrm{O}_{3}$ photoelectrodes

Solution: $1 \mathrm{~mol} \cdot \mathrm{L}^{-1} \mathrm{NaOH}(\mathrm{pH} 13.6)$; potential: $E=0.3 \mathrm{~V}$ (vs SCE); incident light: AM $1.5\left(100 \mathrm{~mW} \cdot \mathrm{cm}^{-2}\right)$. Inset is the proposed equivalent electrical circuit. $R_{\mathrm{s}}$ : solution resistance, $C_{\mathrm{d}}$ : electric double-layer capacitor, $R_{\mathrm{ct}}$ : charge-transfer resistance, $R_{\mathrm{a}}$ : adsorption resistance, CPE: constant phase element
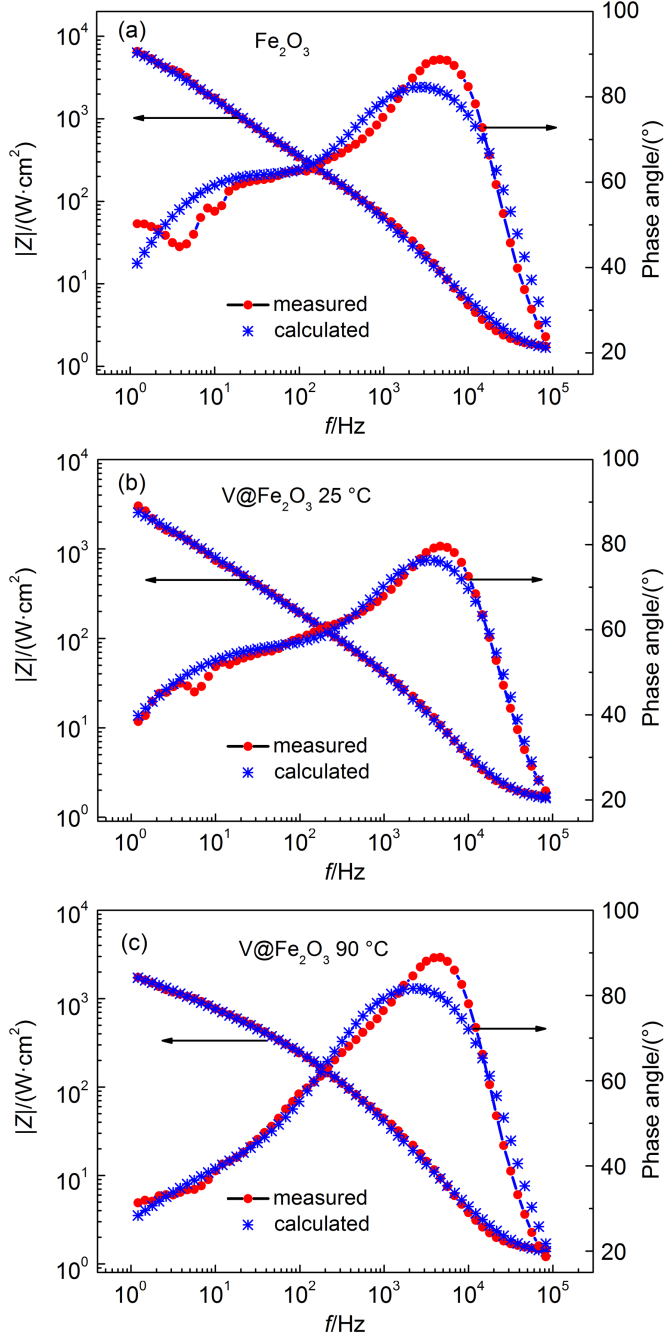

图6 测量与计算的 $\mathrm{Fe}_{2} \mathrm{O}_{3}$ 和钒修饰 $\mathrm{Fe}_{2} \mathrm{O}_{3}$ 光电极的Bode 频谱图

Fig.6 Measured and calculated Bode plots of $\mathrm{Fe}_{2} \mathrm{O}_{3}$ and $\mathrm{V}$-modified $\mathrm{Fe}_{2} \mathrm{O}_{3}$ photoelectrodes

表2. 由表2可见，钒修饰 $\mathrm{Fe}_{2} \mathrm{O}_{3}$ 电极的 $R_{\mathrm{cr}}$ 和 $R_{\mathrm{a}}$ 值均明 显小于未修饰 $\mathrm{Fe}_{2} \mathrm{O}_{3}$ 电极的 $R_{\mathrm{ct}}$ 和 $R_{\mathrm{a}}$ 值, 反映出钒修饰 对界面电荷传输过程和光生中间物种吸脱附过程 的改善作用. 电极/溶液界面Faraday电荷传输过程 动力学是决定电极光电流及其大小的内在因素, 该 过程的表观准一级速率常数 $k$ 可由下式确定:

$$
k=\frac{1}{C_{\mathrm{dl}} R_{\mathrm{ct}}}
$$

根据式(6)及 $C_{\mathrm{dl}} R_{\mathrm{ct}}$ 数据, 三种光电极在 $1 \mathrm{~mol} \cdot \mathrm{L}^{-1}$ $\mathrm{NaOH}$ (pH 13.6)溶液中的界面电荷传输过程的准一 级速率常数分别为 $k\left(\mathrm{Fe}_{2} \mathrm{O}_{3}\right)=1302.9 \mathrm{~s}^{-1} 、 k\left(\mathrm{~V} @ \mathrm{Fe}_{2} \mathrm{O}_{3}\right.$ $\left.25^{\circ} \mathrm{C}\right)=1467.1 \mathrm{~s}^{-1} 、 k\left(\mathrm{~V} @ \mathrm{Fe}_{2} \mathrm{O}_{3} 90^{\circ} \mathrm{C}\right)=2966.2 \mathrm{~s}^{-1}$ (表2). 该结果与分别利用三种电极所测量到的光电 
表2 光电极材料的EIS 等效电路元件参数

Table 2 Parameters of EIS equivalent electrical elements for the photoelectrode materials

\begin{tabular}{cccccccc}
\hline Material & $R_{\mathrm{s}} /\left(\Omega \cdot \mathrm{cm}^{2}\right)$ & $10^{6} \mathrm{C}_{\mathrm{d} /} /\left(\mathrm{F} \cdot \mathrm{cm}^{-2}\right)$ & $R_{\mathrm{cl}} /\left(\Omega \cdot \mathrm{cm}^{2}\right)$ & $Y_{0} /\left(\mathrm{s}^{n} \cdot \Omega^{-1} \cdot \mathrm{cm}^{-2}\right)$ & $n$ & $R^{\mathrm{a}} /\left(\Omega \cdot \mathrm{cm}^{2}\right)$ & $k^{\dagger} / \mathrm{s}^{-1}$ \\
\hline $\mathrm{Fe}_{2} \mathrm{O}_{3}$ & 1.49 & 2.50 & 307.0 & $2.422 \times 10^{-5}$ & 0.707 & 13180 & 1302.9 \\
$\mathrm{~V} @ \mathrm{Fe}_{2} \mathrm{O}_{3} 25^{\circ} \mathrm{C}$ & 1.53 & 3.32 & 205.3 & $7.943 \times 10^{-5}$ & 0.630 & 6799 & 1467.1 \\
$\mathrm{~V} @ \mathrm{Fe}_{2} \mathrm{O}_{3} 90^{\circ} \mathrm{C}$ & 1.29 & 3.75 & 89.9 & $1.474 \times 10^{-4}$ & 0.520 & 3784 & 2966.2 \\
\hline
\end{tabular}

${ }^{\dagger}$ estimated according to Eq.(6); $k$ : pseudo-first-order rate constant

流大小(图3、图4)较好地吻合. 因此，钒修饰对 $\mathrm{Fe}_{2} \mathrm{O}_{3}$ 材料光电化学活性的增强作用, 可归因于钒修 饰对“光电极/溶液”界面处光生电荷界面传输动力 学过程及性能的改善. 同时, 在较高温度 $\left(90^{\circ} \mathrm{C}\right)$ 下 所制备的钒修饰 $\mathrm{Fe}_{2} \mathrm{O}_{3}$ 电极界面电荷传输速率高于 在较低温度 $\left(25^{\circ} \mathrm{C}\right)$ 下所制备的钒修饰 $\mathrm{Fe}_{2} \mathrm{O}_{3}$ 电极, 其 原因可能是较高的温度更有利于钒离子物种与 $\mathrm{Fe}_{2} \mathrm{O}_{3}$ 表面发生物理化学相互作用, 从而使材料表面 修饰的 $\mathrm{FeVO}_{4}$ 晶相结构更为完整(图1(c)), 对阳极析 氧反应的光电催化活性更高.

界面电荷传递速率常数数值的大小与光照条 件、电极材料、施加电位、测量方法及理论模型 等因素有关..$^{11,38,50}$ 最近, Bertoluzzi和Bisquert ${ }^{52}$ 针对 “半导体/溶液”界面的PEC水分解反应, 提出了一个 一般化的EIS动力学物理模型, 不同情况下半导体 光电极表面电荷传输过程的一级速率常数 $\left(k_{\mathrm{s}}\right)$ 的典 型值为 $10^{1}-10^{3} \mathrm{~s}^{-1}$ 数量级 (参见文献 ${ }^{52}$ 中的图 3 和图 5). Peter与其合作者 ${ }^{20,50}$ 分别利用EIS和强度调制光电流 谱(IMPS)技术, 研究了 $\mathrm{Fe}_{2} \mathrm{O}_{3}$ 和钴修饰 $\mathrm{Fe}_{2} \mathrm{O}_{3}$ 光阳极表 面析氧动力学(入射光波长 $\lambda=455 \mathrm{~nm}, 1 \mathrm{~mol} \cdot \mathrm{L}^{-1}$ $\mathrm{NaOH}$ 溶液中), 所确定的电荷传输过程的一级速率 常数 $\left(k_{\mathrm{r}}\right)$ 的数值为 $10^{0}-10^{2} \mathrm{~s}^{-1}$. 这些结果与本文所确定 的一级速率常数 $(k)$ 的数值相近 (虽然不同作者所使 用的理论模型不同).

\section{4 “光电极/溶液”界面能级模型}

图 7 是结合有关研究结果构建的“光电极/1 $\mathrm{mol} \cdot \mathrm{L}^{-1} \mathrm{NaOH}$ 溶液” 界面研究体系的能级模型, 电 位标度以标准氢电极 $(\mathrm{NHE})$ 为基准 $(E(v S \mathrm{SCE})=$ $+0.24 \mathrm{~V}(v s \mathrm{NHE}))$. 在 $1 \mathrm{~mol} \cdot \mathrm{L}^{-1} \mathrm{NaOH}(\mathrm{pH}$ 13.6) 溶 液中, $\mathrm{Fe}_{2} \mathrm{O}_{3}$ 半导体材料的导带 $(\mathrm{CB})$ 底电位 $E_{\mathrm{CB}}$ 约为 $-0.5 \mathrm{~V}(v s \mathrm{NHE}){ }^{2} E_{\mathrm{g}}=2.05 \mathrm{eV}$ (图2(b)), 故其价带 $(\mathrm{VB})$ 顶电位 $E_{\mathrm{VB}}$ 为 $+1.55 \mathrm{~V}$ ( vs NHE), $\mathrm{Fe}_{2} \mathrm{O}_{3}$ 在平带条 件下的能带结构如图7(a)所示. 另外, 由图3可以看 出, 在光照条件下, 钒修饰 $\mathrm{Fe}_{2} \mathrm{O}_{3}$ 电极与未修饰 $\mathrm{Fe}_{2} \mathrm{O}_{3}$ 电极的光电流起始电位基本相同 $\left(E_{\mathrm{on}}=(-0.18 \pm\right.$ $0.02) \mathrm{V}(v s \mathrm{SCE})$ ), 故可认为钒修饰对电极平带电位

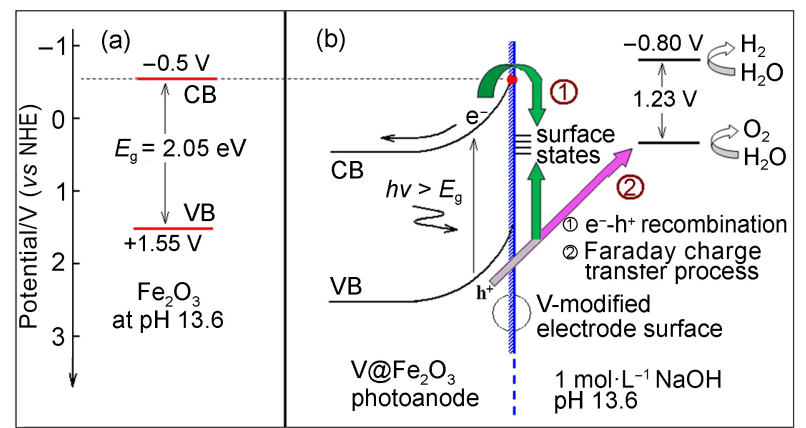

图7 研究体系的能带模型及“光电极/溶液”界面电荷传输 过程示意图

Fig.7 Schematic depiction of band energy level and interfacial charge transfer for the "photoelectrode/solution" interface under investigation

The potential scale is normal hydrogen electrode (NHE), and that the surface-state-mediated charge recombination (process (1) and the charge transfer for $\mathrm{O}_{2}$ evolution (process (2)) compete with each other for capturing photoholes $\left(\mathrm{h}^{+}\right)$.

的影响很小(可以忽略). “光电极/溶液”界面在光照 及阳极极化条件下的能带结构如图7(b)所示. 此时 $\mathrm{Fe}_{2} \mathrm{O}_{3}$ 能带发生弯曲, 光生载流子在“电极/溶液”界面 (或界面区)发生两个过程(图7(b)). 一方面, 光生导 带电子在空间电荷区表面电场作用下流向半导体 内部, 并通过外电路流向Pt阴极发生析氢反应; 光生 价带空穴则穿过 “光电极/溶液”界面向溶液一侧转 移, 在光电极表面引起阳极析氧反应(即过程(2), 图 7(b)):

$$
\mathrm{h}^{+}(\mathrm{VB})+\frac{1}{2} \mathrm{H}_{2} \mathrm{O} \rightarrow \frac{1}{4} \mathrm{O}_{2}+\mathrm{H}^{+}
$$

另一方面, 一部分空穴将陷入能级位于禁带中的表 面态, ${ }^{11,36,51}$ 并通过表面态与导带电子发生表面复合 (即过程(1), 图7(b)):

$$
\mathrm{h}^{+}(\mathrm{VB})+\mathrm{e}^{-}(\mathrm{CB}) \rightarrow \text { null }
$$

过程(1)与过程(2)相互竞争, 共同捕获价带空 穴. ${ }^{20,36}$ 光生空穴向溶液一侧的传递速率越快, 则表 面复合几率越小, 通过外电路检测到的光电流响应 越大. 因此可以认为, 由于钒对 $\mathrm{Fe}_{2} \mathrm{O}_{3}$ 的表面修饰改 
善了电极/溶液界面电荷传输性能, 钒修饰电极 “V@ $\mathrm{Fe}_{2} \mathrm{O}_{3} 90{ }^{\circ} \mathrm{C}$ ”的准一级速率常数是 $\mathrm{Fe}_{2} \mathrm{O}_{3}$ 电极准 一级速率常数的 2.28 倍(表 2 ), 从而使光生电荷在电 极表面的复合几率SPR显著降低(表1), 电极的光电 流密度明显增大(图3、图4), 这是钒修饰使 $\mathrm{Fe}_{2} \mathrm{O}_{3}$ 材 料光电化学活性增强的原因.

另外值得注意的是, 矾酸盐(包括 $\mathrm{FeVO}_{4}$ ) 是一类 新型的 $n$ 型半导体光催化剂, $\mathrm{FeVO}_{4}$ 禁带宽度 $E_{\mathrm{g}}$ 为 2-2.2 eV (与 $\mathrm{Fe}_{2} \mathrm{O}_{3}$ 相近), ${ }^{53-55}$ 具有可见光活性. 近期 研究表明, $\mathrm{FeVO}_{4}$ 或 $\mathrm{FeVO}_{4}-\mathrm{Fe}_{2} \mathrm{O}_{3}$ 二元体系不仅在光 照下可用于环境有机污染物的氧化降解, ${ }^{33,55-57}$ 而且 在无光照条件下对有机物 (如亚甲基蓝、苯、甲 苯、醇类分子等)的化学氧化反应具有显著的催化 $\left(\mathrm{FeVO}_{4}\right)$ 与协同催化 $\left(\mathrm{FeVO}_{4}-\mathrm{Fe}_{2} \mathrm{O}_{3}\right)$ 作用..$^{31,34,58-60}$ 这些 结果与本文所观察到的钒修饰对 $\mathrm{Fe}_{2} \mathrm{O}_{3}$ 表面水的 PEC氧化分解反应的促进作用具有一定的相似性和 一致性.

\section{4 结 论}

利用XRD、UV-Vis-DRS、光电流测量和交流 阻抗实验技术, 研究了钒修饰对 $\alpha-\mathrm{Fe}_{2} \mathrm{O}_{3}$ 光电极材料 光电化学性能的增强作用及原因. 基于本文实验结 果得到以下结论:

(1) 结合水热化学、室温 $\left(25^{\circ} \mathrm{C}\right)$ 和 $90{ }^{\circ} \mathrm{C} \mathrm{VOSO}_{4}$ 溶液化学池沉积 $\left(25\right.$ 和 $90{ }^{\circ} \mathrm{C} \mathrm{VOSO}$ 溶液中) 和后续 $550{ }^{\circ} \mathrm{C}$ 热处理等方法, 分别制备了未修饰 $\mathrm{Fe}_{2} \mathrm{O}_{3}$ 和 2 种钒修饰 $\mathrm{Fe}_{2} \mathrm{O}_{3}$ 光电极材料; XRD和UV-Vis-DRS结 果表明, 钒修饰 $\alpha-\mathrm{Fe}_{2} \mathrm{O}_{3}$ 样品表面存在正交结构的 $\mathrm{FeVO}_{4}$ 晶相, 但钒修饰对材料的可见光吸收性能无 明显影响;

(2) 在 $1 \mathrm{~mol} \cdot \mathrm{L}^{-1} \mathrm{NaOH}(\mathrm{pH}$ 13.6) 中进行的光电 化学测量表明, 钒修饰 $\mathrm{Fe}_{2} \mathrm{O}_{3}$ 电极材料的光电流显著 高于未修饰的 $\mathrm{Fe}_{2} \mathrm{O}_{3}$ 材料, 且在 $90{ }^{\circ} \mathrm{C} \mathrm{VOSO}_{4}$ 溶液中 制备的钒修饰 $\mathrm{Fe}_{2} \mathrm{O}_{3}$ 电极的光电化学活性高于在室 温 $25{ }^{\circ} \mathrm{C} \mathrm{VOSO}_{4}$ 溶液中制备的钒修饰 $\mathrm{Fe}_{2} \mathrm{O}_{3}$ 电极; 经 过钒修饰后, 光生载流子在“电极/溶液”界面处的电 荷复合几率显著降低(约为未修饰 $\mathrm{Fe}_{2} \mathrm{O}_{3}$ 电极的 $1 / 4-1 / 5)$;

(3) 利用交流阻抗谱测量研究了“光电极/溶液” 界面处的Faraday电荷传输性能, 结果表明, 表面钒 修饰对 $\alpha-\mathrm{Fe}_{2} \mathrm{O}_{3}$ 材料光电化学性能的增强作用以及 对光生电荷表面复合过程的抑制作用, 可归因于钒 修饰对“光电极/溶液”界面电荷传输性能的改善与
增强作用, 钒修饰电极“ $\mathrm{V} @ \mathrm{Fe}_{2} \mathrm{O}_{3} 90{ }^{\circ} \mathrm{C}$ ”和未修饰 $\mathrm{Fe}_{2} \mathrm{O}_{3}$ 电极的界面电荷传输准一级速率常数分别为 $k\left(\mathrm{~V} @ \mathrm{Fe}_{2} \mathrm{O}_{3} 90^{\circ} \mathrm{C}\right)=2966.2 \mathrm{~s}^{-1} 、 k\left(\mathrm{Fe}_{2} \mathrm{O}_{3}\right)=1302.9 \mathrm{~s}^{-1}$; 结合 $\mathrm{Fe}_{2} \mathrm{O}_{3}$ /溶液界面能带模型, 分析了Faraday电荷 传输与表面复合过程对研究体系光电化学相应的 影响.

\section{References}

(1) Yang, X.; Liu, R.; He, Y.; Thorne, J.; Zheng, Z.; Wang, D. Nano Res. 2015, 8, 56. doi: 10.1007/s12274-014-0645-2

(2) van de Krol, R.; Grätzel, M. Introduction, Principles of Photoelectrochemical Cells. In Photoelectrochemical Hydrogen Production; van de Krol, R., Grätzel, M. Eds.; Springer Science + Business Media: New York, 2012; pp 3-67.

(3) Valdés, A.; Brillet, J.; Grätzel, M.; Gudmundsdóttir, H.; Hansen, H. A.; Jónsson, H.; Klüpfel, P.; Kroes, G. J.; Le Formal, F.; Man, I. C.; Martins, R. S.; Nørskov, J. K.; Rossmeisl, J.; Sivula, K.; Vojvodic, A.; Zäch, M. Phys. Chem. Chem. Phys. 2012, 14, 49. doi: $10.1039 / \mathrm{C} 1 \mathrm{CP} 23212 \mathrm{~F}$

(4) Chen, X.; Shen, S.; Guo, L.; Mao, S. S. Chem. Rev. 2010, 110, 6503. doi: $10.1021 / \mathrm{cr} 1001645$

(5) Zhou, W.; Xie Q.; Lian, S. Prog. Chem. 2013, 25, 1989. [周文 理, 谢青季, 廉世勋. 化学进展, 2013, 25, 1989.]

(6) Diab, M.; Mokari, T. Inorg. Chem. 2014, 53. 2304.

(7) Rangaraju, R. R.; Raja, K. S.; Panday, A.; Misra, M. Electrochim. Acta 2010, 55, 785. doi: 10.1016/j.electacta.2009.07.012

(8) Pradhan, G. K.; Padhi, D. K.; Parida, K. M. ACS Appl. Mater. Interfaces 2013, 5, 9101. doi: 10.1021/am402487h

(9) Lee, C. Y.; Wang, L.; Kado, Y.; Kirchgeorg, R.; Schmuki, P. Electrochem. Commun. 2013, 34, 308. doi: 10.1016/j.elecom.2013.07.024

(10) Wang, L.; Lee, C.Y.; Schmuki, P. Electrochem. Commun. 2013, 30, 21. doi: 10.1016/j.elecom.2013.01.013

(11) Shangguan, P. P.; Tong, S. P.; Li, H. L.; Leng, W. H. Acta Phys. -Chim. Sin. 2013, 29, 1954. [上官鹏鹏, 童少平, 李海丽, 冷文华. 物理化学学报, 2013, 29, 1954.] doi: 10.3866/PKU.WHXB201306261

(12) Jin, H.; Wang, J.; Ji, Y.; Chen, M. M.; Zhang, Y.; Wang, C.; Cong, Y. Q. Acta Phys.-Chim. Sin. 2015, 31, 955. [金 环, 王 娟, 姬 云, 陈媚媚, 张 轶, 王 齐, 从燕青. 物理化学学 报, 2015, 31, 955.] doi: 10.3866/PKU.WHXB201503112

(13) Kumar, P.; Sharma, P.; Shrivastav, R.; Dass, S.; Satsangi, V. R. Int. J. Hydrog. Energy 2011, 36, 2777. doi: 10.1016/j.ijhydene.2010.11.107

(14) Zhang, X.; Li, H.; Wang, S.; Fan, F. R. F.; Bard, A. J. J. Phys. Chem. C 2014, 118, 16842. doi: 10.1021/jp500395a

(15) Shaban, Y. A.; Khan, S. U. M. Sci. Adv. Mater. 2012, 4, 356. doi: 10.1166/sam.2012.1292

(16) Barroso, M.; Mesa, C. A.; Pendlebury, S. R.; Cowan, A. J.; 
Hisatomi, T.; Sivula, K.; Grätzel, M.; Klug, D. R.; Durrant, J. R. PNAS 2012, 109, 15640. doi: 10.1073/pnas.1118326109

(17) Barroso, M.; Cowan, A. J.; Pendlebury, S. R.; Grätzel, M.; Klug, D. R.; Durrant, J. R. J. Am. Chem. Soc. 2011, 133, 14868. doi: 10.1021/ja205325v

(18) Klahr, B.; Gimenez, S.; Fabregat-Santiago, F.; Bisquert, J.; Hamann, T. W. J. Am. Chem. Soc. 2012, 134, 16693. doi: 10.1021/ja306427f

(19) Shen, S.; Zhou, J.; Dong, C. L.; Hu, Y.; Tseng, E. N.; Guo, P.; Guo, L.; Mao, S. S. Sci. Rep. 2014, 4, 6627. doi: 10.1038/srep06627

(20) Peter, L. M.; Wijayantha, K. G. U.; Tahir, A. A. Faraday Discuss. 2012, 155, 309. doi: 10.1039/C1FD00079A

(21) Sun, W.; Meng, Q.; Jing, L.; Liu, D.; Cao, Y. J. Phys. Chem. C 2013, 117, 1358. doi: 10.1021/jp309599d

(22) Augustynski, J.; Alexander, B. D.; Solarska, R. Top. Curr. Chem. 2011, 303, 1. doi: 10.1007/978-3-642-22294-8

(23) Varshney, D.; Yogi, A. J. Mol. Struct. 2011, 995, 157. doi: 10.1016/j.molstruc.2011.04.011

(24) Martis, V.; Oldman, R.; Anderson, R.; Fowles, M.; Hyde, T.; Smith, R.; Nikitenko, S.; Bras, W.; Sankar, G. Phys. Chem. Chem. Phys. 2013, 15, 168. doi: 10.1039/C2CP43307A

(25) Sun, L.; Wu, W.; Yang, S.; Zhou, J.; Hong, M.; Xiao, X.; Ren, F.; Jiang, C. ACS Appl. Mater. Interfaces 2014, 6, 1113. doi 10.1021/am404700h

(26) Zhou, W.; Li, T.; Wang, J.; Qu, Y.; Pan, K.; Xie, Y.; Tian, G.; Wang, L.; Ren, Z.; Jiang, B.; Fu, H. Nano Res. 2014, 7, 731. doi: 10.1007/s12274-014-0434-y

(27) Jana, S.; Mondal, A. ACS Appl. Mater. Interfaces 2014, 6, 15832. doi: 10.1021/am5030879

(28) Huang, Y. C.; Zhao, Z. F.; Li, S. X.; D, J.; Zheng, H. J. Chin. J. Inorg. Chem. 2015, 31, 133. [黄益操, 赵浙菲, 李世雄, 邸 婧, 郑华均. 无机化学学报, 2015, 31, 133.]

(29) Xu, Z.; Huang, C.; Wang, L.; Pan, X.; Qin, L.; Guo, X.; Zhang, G. Ind. Eng. Chem. Res. 2015, 54, 4593. doi: 10.1021/acs.iecr.5b00335

(30) Mohapatra, S. K.; Banerjee, S.; Misra, M. Nanotechnology 2008, 19, 315601. doi: 10.1088/0957-4484/19/31/315601

(31) Oliveira, H. S.; Oliveira, L. C. A.; Pereira, M. C.; Ardisson, J. D.; Souza, P. P.; Patricio, P. O.; Moura, F. C. C. New J. Chem. 2015, 39, 3051. doi: 10.1039/C4NJ02063D

(32) Hwang, H. K.; Seo, J. W.; Seo, W. S.; Lim, Y. S.; Park, K. Int. J. Energy Res. 2014, 38, 241. doi: 10.1002/er.v38.2

(33) Wang, M.; Wang, L. A.; Zhou, L. N.; Zhang, W. J. J. Chin. Ceram. Soc. 2009, 37, 203. [王 敏, 王里奥, 周丽娜, 张文杰. 硅酸盐学报, 2009, 37, 203.]

(34) Zhang, G. Q.; Zhang, X.; Lin, T.; Gong, T.; Qi, M. Chin. Chem. Lett. 2012, 23, 145. doi: 10.1016/j.cclet.2011.10.015

(35) Zhang, G. Q.; Zhang, X.; Qi, M.; Lin, T.; Gong, T. Chin. J. Catal. 2012, 33, 870. [张贵泉, 张 昕, 祁 敏, 林 涛, 龚＼cjkstart婷. 催化学报, 2012, 33, 870.]
(36) Kong, D. S.; Wei, Y. J.; Li, X. X.; Zhang, Y.; Feng, Y. Y.; Li, W. J. J. Electrochem. Soc. 2014, 161, H144.

(37) Kong, D. S.; Zhang, X. D.; Wang, J.; Wang, C.; Zhao, X.; Feng, Y. Y.; Li, W. J. J. Solid State Electrochem. 2013, 17, 69. doi: 10.1007/s10008-012-1854-9

(38) Zhang, J. W.; Kong, D. S.; Zhang, H.; Du, D. D.; Wang, N.; Feng, Y. Y.; Li, W. J. J. Solid State Electrochem. 2015, accepted. doi: 10.1007/s10008-015-2948-y

(39) Kong, D. S. Langmuir 2010, 26, 4880. doi: 10.1021/la9036869

(40) Li, W. J.; Du, D. D.; Yan, T. J.; Kong, D. S.; You, J. M.; Li, D. Z. J. Colloid Interface Sci. 2015, 444, 42. doi: 10.1016/j.jcis.2014.12.052

(41) Du, D. D; Li, W. J.; Chen, S. S.; Yan, T. J.; You, J. M.; Kong, D. S. New J. Chem. 2015, 39, 3129.

(42) Li, W. J.; Kong, D. S.; Cui, X. L.; Du, D. D.; Yan, T. J.; You, J. M. Mater. Res. Bull. 2014, 51, 69. doi: 10.1016/j.materresbull.2013.12.007

(43) ZSimpWim Echem Software, Version 3.20d, 2004; Jade 5.0 (MDI) software package, 2004.

(44) Barron, V.; Torrent, J. J. Soil Sci. 1986, 37, 499. doi: 10.1111/ejs.1986.37.issue-4

(45) Li, W.; Li, D.; Chen, Z.; Huang, H.; Sun, M.; He, Y.; Fu, X. J. Phys. Chem. C 2008, 112, 14943. doi: 10.1021/jp8049075

(46) Boudjemaa, A.; Boumaza, S.; Trari, M.; Bouarab, R.; Bouguelia, A. Int. J. Hydrog. Energy 2009, 34, 4268. doi: 10.1016/j.ijhydene.2009.03.044

(47) de Tacconi, N. R.; Boyles, C. A.; Rajeshwar, K. Langmuir 2000, 16, 5665. doi: 10.1021/la000037x

(48) Radecka, M.; Wierzbicka, M.; Komornicki, S.; Rekas, M. Phys. B 2004, 348, 160. doi: 10.1016/j.physb.2003.11.086

(49) Bonanos, N.; Steele, B. C. H.; Butler, E. P.; MacDonald, J. R.; Johnson, W. B.; Worrell, W. L.; MacDonald, D. D.; McKubre, M. C. H.; Barsoukov, E.; Conway, B. E.; Wagner, N. Applications of Impedance Spectroscopy. In Impedance Spectroscopy: Theory, Experiment, and Applications, 2nd ed.; Barsoukov, E., MacDonald, J. R. Eds.; John Wiley \& Sons, Inc.: New Jersey, 2005; pp 205-537.

(50) Wijayantha, K. G. U.; Saremi-Yarahmadi, S.; Peter, L. M. Phys. Chem. Chem. Phys. 2011, 13, 5264. doi: 10.1039/c0cp02408b

(51) Klahr, B.; Gimenez, S.; Fabregat-Santiago, F.; Hamann, T.; Bisquertm, J. J. Am. Chem. Soc. 2012, 134, 4294. doi: 10.1021/ja210755h

(52) Bertoluzzi, L.; Bisquert, J. J. Phys. Chem. Lett. 2012, 3 , 2517. doi: 10.1021/jz3010909

(53) Rao, N. S.; Palanna, O. G. Bull. Mater. Sci. 1995, 18, 229. doi: 10.1007/BF02749660

(54) Li, A. T.; Cao, L. Y.; Huang, J. F.; Huang, Y. C.; Wu, J. P. J. Synth. Cryst. 2012, 41, 1227. [李阿婷, 曹丽云, 黄剑锋, 黄毅成, 吴建鹏. 人工晶体学报, 2012, 41, 1227.]

(55) Wang, M.; Luan, H. Y.; Yu, P.; Che, Y. S.; Niu, C.; Dong, D. Chin. J. Nonferrous Metals 2013, 23, 2243. [王 敏, 奕海燕, 
余 萍, 车寅生, 牛 超, 董 多. 中国有色金属学报, 2013, 23, 2243.] doi: 10.1016/S1003-6326(13)62724-7

(56) Rao, Z.; Gu, Y.; Huang, C. Y.; He, Y.; Huang, Y. P.; Zhang, A. Q. Environ. Chem. 2013, 32, 564. [ 饶 志, 顾 彦, 黄春迎, 何 燕, 黄应平, 张爱清. 环境化学, 2013, 32, 564.].

(57) Liu, Y.; Dai, C. H.; Ma, J. F.; Song, Z. W.; Sun, Y.; Fang, J. R.; Zgao, J. G.; Sun, X.; Gao, C.; Liu, Z. S. Bull. Chin. Ceram. Soc. 2008, 28, 1220. [刘 晔, 戴长虹, 马峻峰, 宋祖伟, 孙 勇, 房
晶瑞, 赵金刚, 孙 霞, 高 敞, 刘振森. 硅酸盐通报, 2008, 28, 1220.]

(58) Liang, X.; Zhu, S.; Zhong, Y.; Zhu, J.; Yuan, P.; He, H.; Zhang, J. Appl. Catal. B 2010, 97, 151. doi: 10.1016/j.apcatb.2010.03.035

(59) Kaneti, Y. V.; Zhang, Z.; Yue. J.; Jiang, X.; Yu, A. J. Nanopart. Res. 2013, 15, 1948. doi: 10.1007/s11051-013-1948-Z

(60) Kim, K.; Kim, I. H.; Yoon, K. Y.; Lee, J.; Jang, J. H. J. Mater Chem. A 2015, 3, 7706. doi: 10.1039/C5TA00027K

\section{欢迎订阅《大学化学》}

经北京市新闻出版广电局批复(京新广函 [2015］200号), 自2016年1月起: 《大学化学》(CN11-1815/O6)由双月刊变更 为月刊。

《大学化学》是由教育部主管、北京大学和中国化学会共同主办的化学教育类刊物。主要介绍化学科学的新进展, 开展 与教学有关的重大课题的研讨, 交流教学改革经验, 报道化学及其相关学科的新知识、新动向, 促进教师知识更新, 扩大 学生知识面, 为提高教学水平服务。主要栏目有: 今日化学、教学研究与改革、知识介绍、化学实验、师生笔谈、自学之 友、大学化学先修课程、竞赛园地、国外化学教育、化学史、书评以及专题讨论等。

2016年每本定价12.00元, 全年出版12期, 共144.00元。

全国各地邮局均可订阅, 邮发代号：82-314。为方便读者订阅, 本刊编辑部全年办理邮购业务。

邮购地址: 北京大学化学学院 《大学化学》编辑部

邮编: 100871

电话: 010-62751721

Email: dxhx@pku.edu.cn

网址: http://www.dxhx.pku.edu.cn 